\title{
LES MOTS DE LA TÊTE. LES LEXÈMES DÉSIGNANT LA TÊTE EN LANGUE POPULAIRE / ARGOTIQUE DU XIX ${ }^{E}$ SIÈCLE
}

\author{
Jean-Pierre GOUDAILLIER \\ Université de Paris, Paris
}

\begin{abstract}
En): The lexemes cafetière, fiole and Sorbonne have one thing in common: they all designate the head in popular language and / or in slang. By the shift of meaning, there are many terms used with a non-standard linguistic value and they constitute an important lexical stock for the "head" paradigm. The Dictionary of Slang by Jean-Paul COLIN \& Jean-Pierre MÉVEL, contains 64 slang terms to designate the head in the 20th century. 32 / 64 lexemes have been selected for this work analysis. 17 glossaries and dictionaries published between 1827 and 1907 were selected because they include, among their entries, one or more of the 32 slang lexemes designating the head. For the 32 terms their etymology and their dating were established, as well as the formal and semantic procedures that were used for their formation. The analysis of all the nouns offers the possibility to determine those that have remained or have simply disappeared from contemporary uses of popular language and / or slang. It is the substantive tronche that seems to be the most used in the 19th century, because it constitutes an entry in the majority of retained glossaries and dictionaries. Sorbonne is in second position.
\end{abstract}

Keywords (En): 19th century; slang; dictionaries; etymology; slang lexemes; formal procedures; semantic procedures

Mots-clés (Fr) : XIX ${ }^{\mathrm{e}}$ siècle ; argot ; dictionnaires ; étymologie ; lexèmes d'argot ; procédés formels ; procédés sémantiques

DOI : $10.32725 /$ eer.2021.005

\section{Introduction}

Les lexèmes cafetière, fiole et Sorbonne ont un point commun; tous les trois désignent la tête en langue populaire et / ou argotique. Par glissement de sens, de nombreux termes sont ainsi utilisés avec un sens figuré, une valeur linguistique nonstandard et constituent de ce fait un stock lexical pour le paradigme «tête ». Dans son édition de 1990, Le Dictionnaire de l'argot de Jean-Paul Colin \& Jean-Pierre Mével relève 64 lexèmes signifiant «tête ». Pour un certain nombre de ces termes, à savoir parmi les plus usités, il s'avère intéressant, à partir de ce dictionnaire et en utilisant aussi des dictionnaires et glossaires datant essentiellement de la deuxième moitié du XIX ${ }^{\mathrm{e}}$ siècle et du début du $\mathrm{XX}^{\mathrm{e}}$ siècle, d'établir leur datation, ainsi que les procédés formels et / ou sémantiques ayant été utilisés pour leur formation. Il peut en être de même pour d'autres mots non répertoriés par Jean-Paul Colin \& JeanPierre Mével.

\section{Méthodologie}

À partir du Dictionnaire de l'argot de Jean-Paul Colin \& Jean-Pierre Mével, qui témoigne de l'emploi de 64 termes pour désigner la tête au $\mathrm{XX}^{\mathrm{e}}$ siècle, ont été 
retenus 32 lexèmes, une fois éliminées les formes similaires renvoyant à un seul et même mot, comme tranche, tronche. Un certain nombre de doublets du type de cabèche, cabochon, voire des éléments faisant partie de séries telles bobe, bobine, bobinasse, bobinette, bobèche, bobéchon, etc. ont été aussi retenus, lorsque leur fréquence d'utilisation est importante : ainsi, bobine est toujours assez utilisée, ce qui n'est plus le cas de bobe, bobinasse, bobinette.

Durant le XIX ${ }^{\mathrm{e}}$ siècle paraît un nombre relativement important de dictionnaires et glossaires d'argot notables. Pour cette étude ont été sélectionnés 17 d'entre eux parus entre 1827 et 1907, qui offrent au total environ 34000 entrées constituant un aperçu de l'évolution de l'argot dit classique au cours du XIX ${ }^{\mathrm{e}}$ siècle. Ces 17 glossaires et dictionnaires ont été sélectionnés, car ils comportent parmi leurs entrées un ou plusieurs des 32 lexèmes désignant la tête retenus du fait de la méthodologie appliquée.

\section{Dictionnaires contenant des lexèmes désignant la tête en langue populaire/argotique au $\mathrm{XIX}^{\mathrm{e}}$ siècle retenus pour cette étude}

Compte tenu de ce qui a été indiqué ci-dessus en 2. les 17 dictionnaires retenus sont dans l'ordre chronologique croissant :

01) Dictionnaire d'argot ou Guide des gens du monde, pour les tenir en garde contre les mouchards, filous, filles de joie, et autres fashionables et petitesmaîtresses de la même trempe. Par un monsieur comme il faut, ex-pensionnaire de Ste-Pélagie par un anonyme, paru en 1827 ;

02) « Glossaire d'argot», in : Mémoires d'un forçat ou Vidocq dévoilé (tome quatrième) de Louis François Raban \& Émile Marco de Saint-Hilaire datant de 1829 ;

03) Nouveau dictionnaire d'argot de Bras-de-Fer édité en 1829 ;

04) Dictionnaire d'argot, in: La Légende noire du bagne de Jean-Joseph Clémens datant de 1840 ;

05) Dictionnaire complet de l'Argot employé dans les Mystères de Paris. Ouvrage éminemment utile à toute personne honnête puisqu'il divulgue à la société les mots dont les filoux, voleurs, floueurs, chevaliers d'industrie composent leur conversation (ouvrage recueilli par M.D.) paru en 1844 ;

06) Dictionnaire renfermant les mots les plus usités dans le langage des prisons par un détenu anonyme, paru en 1846 dans L'intérieur des prisons - Réforme pénitentiaire, système cellulaire, emprisonnement en commun ;

07) Le nouveau dictionnaire complet du Jargon de l'argot d'Arthur Halbert d'Angers daté de 1849 ;

08) Dictionnaire érotique moderne par un professeur de langue verte d'Alfred Delvau, édition de 1864 ;

09) Les Excentricités du langage de Lorédan Larchey, édition de 1865 ;

10) Dictionnaire de la langue verte - Argots parisiens comparés d'Alfred Delvau, édition de 1867, ainsi que le supplément de cet ouvrage de Gustave Fustier daté de 1889 ; 
11) Dictionnaire de l'argot des typographes d'Eugène Boutmy, édition de 1883

12) Dictionnaire de l'argot moderne de Lucien Rigaud édité en 1888 ;

13) La langue verte du troupier - dictionnaire d'argot militaire de Léon Merlin, édition de 1888 ;

14) La langue verte. Dictionnaire d'argot et des principales locutions populaires de Jean LA RUE édité en 1894 ;

15) Dictionnaire d'argot fin-de-siècle de Charles Virmaître datant de 1894 ;

16) Dictionnaire d'argot (ARGOT-FRANÇAIS - FRANÇAIS-ARGOT) de Gustave-Armand ROSSIGNOL de 1901 ;

17) Dictionnaire Argot-Français de Napoléon Hayard paru en $1907^{1}$.

\section{Analyse}

Les 32 termes faisant l'objet de l'analyse sont ardoise, balle, bille, binette, bobe, bobèche, bobéchon, bobine, boule, bourriche, bourrichon, boussole, cabèche, caboche, cafetière, caillou, caisson, calebasse, ciboulot, citron, cocarde, coco, coloquinte, fiole, hure, poire, pomme, sorbonne, tirelire, toupie, trombine, tronche.

Le tableau ci-après récapitule les occurrences des 32 lexèmes argotiques désignant la tête retenus dans les 17 dictionnaires et glossaires. Une lecture dans le sens horizontal permet de voir quels glossaires et dictionnaires ont une de leurs entrées correspondant au lexème mentionné à gauche en bout de ligne ; dans le sens vertical chaque colonne indique, pour chaque numéro $\left(01\right.$ à $\left.17^{2}\right)$ de dictionnaire ou de glossaire inscrit en haut de la colonne, les lexèmes signifiant « tête » mentionnés. Ainsi, exemple parmi d'autres, le mot coloquinte se trouve dans 6 des 17 dictionnaires et glossaires retenus pour l'étude, à savoir en 2, 4, 9, 10, 12 et 14 datant respectivement de 1829,1840,1865, 1867, 1888 et $1894^{3}$. Autre exemple : si l'on prend en compte la cinquième édition de l'ouvrage de Lorédan Larchey intitulé Les Excentricités du langage paru en 1865 (colonne 09) on note qu'il contient 6 des 32 termes, à savoir balle, boule, cocarde, coloquinte, sorbonne et tronche, tandis que 17 termes sont présents dans l'édition de 1867 du Dictionnaire de la langue verte d'Alfred Delvau (colonne 10) : bobéchon, bobine, boule, bourrichon, boussole, caboche, caisson, calebasse, cocarde, coco, coloquinte, pomme, sorbonne, tirelire, toupie, trombine et tronche.

Si l'on observe l'ensemble du tableau 1, trois ouvrages, plus particulièrement, retiennent un grand nombre de termes parmi les 32 lexèmes : ce sont dans l'ordre chronologique le Dictionnaire de la langue verte d'Alfred Delvau de 1867 (17 termes), le Dictionnaire de l'argot moderne de Lucien Rigaud de 1888 (23 termes) et La langue verte de Jean La Rue édité en 1894 (19 termes).

Ci-après sont analysées dans le détail les occurrences des 32 termes, avec l'indication le cas échéant de leur morphologie et étymologie, de leur datation.

\footnotetext{
${ }^{1}$ Les deux derniers dictionnaires ont été aussi retenus, bien que datant de 1901 et 1907, car on peut considérer, qu'ils ont été établis à partir de l'exploitation de données linguistiques du XIX ${ }^{\mathrm{e}}$ siècle, tout comme les autres ouvrages utilisés pour cette étude.

${ }^{2} \mathrm{Cf}$. §. 3. pour la liste des 17 ouvrages avec les numéros qui leur correspondent.

${ }^{3}$ Voir note 1.
} 
- ardoise : à partir du sens premier d'ardoise, à savoir 'schiste argileux ... qui se divise en feuillets minces et qu'on utilise à divers usages (principalement couverture des maisons...)' (Tlfi, consulté 06/20), ce lexème désigne en argot par métaphore un couvre-chef (cf. la tuile qui recouvre) et par extension la tête, ce que confirme le Dictionnaire de l'argot moderne (RIGAUD, 1888 : 15). Un deuxième ouvrage parmi les 17 retenus relève ardoise avec le sens de «tête », celui de Jean La Rue (1894).

- balle : construit par analogie de forme par rapport à l'objet de forme sphérique et de petite dimension utilisé dans différents jeux (Tlfi, consulté 06/20), balle est bien un des substantifs relevés en argot pour la tête ('Comme Boule et Coloquinte, balle est une allusion à la rondeur de la tête' (Lorédan LARCHEY, 1865 : 20)) ; une tête bien ronde révélatrice d'un bon état de santé est une bonne balle. Le terme est relevé par Jean-Joseph Clémens dès 1840 et par un troisième ouvrage, celui de Lucien Rigaud (RIGAUD, $1888: 26$ ).

\begin{tabular}{|c|c|c|c|c|c|c|c|c|c|c|c|c|c|c|c|c|c|}
\hline \multicolumn{18}{|l|}{ "tête" } \\
\hline & 1 & 2 & 3 & 4 & 5 & 6 & 7 & 8 & 9 & 10 & 11 & 12 & 13 & 14 & 15 & 16 & 17 \\
\hline & 1827 & 1829 & 1829 & 1840 & 1844 & 1846 & 1849 & 1864 & 1865 & 1867 & 1883 & 1888 & 1888 & 1894 & 1894 & 1901 & 1907 \\
\hline ARDOISE & 0 & 0 & 0 & 0 & 0 & 0 & 0 & 0 & 0 & 0 & 0 & 1 & 0 & 1 & 0 & 0 & 0 \\
\hline BALLE & 0 & 0 & 0 & 1 & 0 & 0 & 0 & 0 & 1 & 0 & 0 & 1 & 0 & 0 & 0 & 0 & 0 \\
\hline BILLE & 0 & 0 & 0 & 1 & 0 & 0 & 0 & 0 & 0 & 0 & 0 & 1 & 0 & 0 & 0 & 0 & 0 \\
\hline BINETTE & 0 & 0 & 0 & 0 & 0 & 0 & 1 & 0 & 0 & 0 & 0 & 1 & 0 & 0 & 0 & 0 & 0 \\
\hline BOBE & 0 & 0 & 0 & 0 & 0 & 0 & 0 & 0 & 0 & 0 & 0 & 1 & 0 & 0 & 0 & 0 & 0 \\
\hline BOBÈCHE & 0 & 0 & 0 & 0 & 0 & 0 & 0 & 0 & 0 & 0 & 0 & 1 & 0 & 0 & 0 & 0 & 0 \\
\hline BOBÉCHON & 0 & 0 & 0 & 0 & 0 & 0 & 0 & 0 & 0 & 1 & 0 & 1 & 0 & 1 & 0 & 0 & 0 \\
\hline BOBINE & 0 & 0 & 0 & 0 & 0 & 0 & 0 & 0 & 0 & 1 & 0 & 1 & 0 & 1 & 0 & 0 & 0 \\
\hline BOULE & 0 & 0 & 0 & 0 & 0 & 0 & 0 & 0 & 1 & 1 & 0 & 1 & 0 & 1 & 0 & 0 & 0 \\
\hline BOURRICHE & 0 & 0 & 0 & 0 & 0 & 1 & 0 & 0 & 0 & 0 & 0 & 0 & 0 & 0 & 0 & 0 & 0 \\
\hline BOURRICHON & 0 & 0 & 0 & 0 & 0 & 0 & 0 & 1 & 0 & 1 & 0 & 1 & 0 & 0 & 0 & 0 & 0 \\
\hline BOUSSOLE & 0 & 0 & 0 & 0 & 0 & 0 & 0 & 0 & 0 & 1 & 0 & 1 & 0 & 1 & 1 & 1 & 1 \\
\hline CABËCHE & 0 & 0 & 0 & 0 & 0 & 0 & 0 & 0 & 0 & 0 & 0 & 0 & 0 & 1 & 0 & 0 & 1 \\
\hline CABOCHE & 0 & 0 & 0 & 0 & 0 & 0 & 1 & 0 & 0 & 1 & 0 & 1 & 0 & 1 & 1 & 1 & 1 \\
\hline CAFETIÈRE & 0 & 0 & 0 & 0 & 0 & 0 & 0 & 0 & 0 & 0 & 0 & 1 & 0 & 1 & 0 & 1 & 0 \\
\hline CAILLOU & 0 & 0 & 0 & 0 & 0 & 0 & 0 & 0 & 0 & 0 & 0 & 0 & 0 & 1 & 1 & 1 & 0 \\
\hline CAISSON & 0 & 0 & 0 & 0 & 0 & 0 & 0 & 0 & 0 & 1 & 0 & 1 & 0 & 1 & 0 & 0 & 0 \\
\hline CALEBASSE & 0 & 1 & 0 & 0 & 0 & 0 & 0 & 0 & 1 & 1 & 0 & 0 & 0 & 1 & 0 & 0 & 0 \\
\hline CIBOULOT & 0 & 0 & 0 & 0 & 0 & 0 & 0 & 0 & 0 & 0 & 0 & 0 & 1 & 1 & 1 & 0 & 0 \\
\hline CITRON & 0 & 0 & 0 & 0 & 0 & 0 & 0 & 0 & 0 & 0 & 0 & 1 & 0 & 0 & 0 & 1 & 1 \\
\hline COCARDE & 0 & 0 & 0 & 0 & 0 & 0 & 0 & 0 & 1 & 1 & 0 & 1 & 0 & 0 & 0 & 0 & 0 \\
\hline COCO & 0 & 0 & 0 & 0 & 0 & 0 & 0 & 0 & 0 & 1 & 0 & 1 & 0 & 0 & 0 & 0 & 0 \\
\hline COLOQUINTE & 0 & 1 & 0 & 1 & 0 & 0 & 0 & 0 & 1 & 1 & 1 & 1 & 0 & 1 & 0 & 0 & 0 \\
\hline FIOLE & 0 & 0 & 0 & 0 & 0 & 0 & 0 & 0 & 0 & 0 & 0 & 1 & 0 & 0 & 0 & 0 & 0 \\
\hline HURE & 0 & 0 & 0 & 0 & 0 & 0 & 0 & 0 & 0 & 0 & 0 & 0 & 0 & 1 & 1 & 1 & 1 \\
\hline POIRE & 0 & 0 & 0 & 0 & 0 & 0 & 0 & 0 & 0 & 0 & 0 & 1 & 0 & 1 & 1 & 1 & 0 \\
\hline POMME & 0 & 0 & 0 & 0 & 0 & 0 & 0 & 0 & 0 & 1 & 0 & 0 & 0 & 1 & 0 & 1 & 0 \\
\hline SORBONNE & 0 & 1 & 1 & 0 & 0 & 0 & 1 & 0 & 1 & 1 & 0 & 1 & 0 & 1 & 1 & 1 & 1 \\
\hline TIRELIRE & 0 & 0 & 0 & 0 & 0 & 0 & 0 & 0 & 0 & 1 & 0 & 1 & 0 & 0 & 1 & 1 & 1 \\
\hline TOUPIE & 0 & 0 & 0 & 0 & 0 & 0 & 0 & 0 & 0 & 1 & 0 & 0 & 0 & 0 & 0 & 0 & 0 \\
\hline TROMBINE & 0 & 0 & 0 & 0 & 0 & 0 & 0 & 0 & 0 & 1 & 0 & 0 & 0 & 1 & 0 & 0 & 0 \\
\hline TRONCHE & 1 & 1 & 1 & 1 & 1 & 1 & 0 & 0 & 1 & 1 & 0 & 1 & 1 & 1 & 1 & 1 & 1 \\
\hline
\end{tabular}

Tableau 1 - Occurrences des lexèmes argotiques désignant la tête dans les 17 dictionnaires

- bille : le même procédé d'analogie de forme que celui utilisé pour balle permet de passer du sens de bille en tant que 'petite boule de pierre peinte ou de verre, servant aux jeux des enfants' (Tlfi, consulté 06 20) à celui de « tête ». Léon Rigaud précise que bille fait partie au départ du jargon des voleurs (1888: 40). Le Tlfi (consulté 06/20) propose comme datation 1881, date de la première édition du 
Dictionnaire de l'argot moderne de Lucien Rigaud. Or, ce terme est relevé dès 1840 par le dictionnaire d'argot de Jean-Joseph Clémens (cf. le tableau 1).

- binette: d'après le Tlfi (consulté 06/20), qui retient comme datation 1836, 'l'hypothèse d'une aphérèse puis d'une dérivation de trombine au sens de "tête ridicule " n'est peut-être pas à écarter'. Des 17 ouvrages retenus Le nouveau dictionnaire complet du Jargon de l'argot d'Arthur Halbert d'Angers est le premier et le seul à citer ce mot (1849: 10), ce qui fournit une datation, qui est d'ailleurs confirmée par le Tlfi (consulté 06/20). Lucien Rigaud (1888:41) ne comporte pas d'entrée et retient ce terme seulement dans l'expression binette à la désastre, tête du créancier impayé.

- bobe: le rapprochement doit être fait avec bobine 4 ('en raison de la forme sphérique de cet objet', précise le Tlfi (consulté 06/20)) et non avec bobèche. Le substantif bobe est noté en 1888 par Lucien Rigaud (1888: 45), une datation crédible étant la plus ancienne pour ce terme (cf. le tableau 1).

- bobèche, bobéchon: une bobèche est une 'pièce cylindrique à rebords, adaptée au chandelier pour recevoir la bougie' (Tlfi, consulté 06/20). Au figuré et dans un usage essentiellement populaire, voire argotique, ce substantif signifie « tête "; le même glissement de sens vers un usage familier, populaire peut être noté pour bobéchon, 'petite bobèche à pointe que l'on peut enfoncer dans le bois ou la pierre' (Tlfi, consulté 06/20). Pour le tlfi la première attestation de bobéchon date de 1866 , ce que confirme le tableau (colonne 10 correspondant au dictionnaire d'Alfred Delvau publié en 1867). En même temps que bobe Lucien Rigaud (1888: 45) note aussi bobèche et bobéchon; si bobe et bobèche ont bien pour datation première 1888 (cf. le tableau 1), ce n'est pas le cas de bobéchon, rappelons-le, déjà relevé par Alfred Delvau en 1867, qui cite la locution se monter le bobêchon ${ }^{5}$, 's'illusionner sur quelqu'un ou sur quelque chose; se promettre monts et merveilles d'une affaire - qui accouche d'une souris' (DELVAU, 1867 : 45).

- bobine : ce substantif désigne en argot la tête par extension du sens d'un terme désignant un 'cylindre de bois, de métal, de matière plastique, muni de rebords, sur lequel on enroule du fil, de la laine ou toute autre matière souple' (Tlfi, consulté en $06 / 20$ ), ce en raison de la forme sphérique de cet objet. Bobine est relevé par Alfred Delvau (1867 : 46), Lucien Rigaud (1888: 45) et Jean La Rue (1894:6).

- boule : pour le Tlfi (consulté 06/20) il s'agit d'un 'objet naturel ou façonné, creux ou plein, de forme sphérique'. En langue argotique le terme est utilisé par analogie de forme pour désigner la «tête». D'un point de vue étymologique le Tlfi retient la datation 1799 par renvoi au chapitre XIV (Dictionnaire d'argot, ou langage des voleurs) d'Histoire des brigands, chauffeurs et assassins d'Orgères de P. Leclair.

Il s'agit de noter que pour balle, bille, binette, bobe, bobèche, bobéchon, bobine et boule référence est faite dans chaque cas à la forme ronde de l'objet désigné pour obtenir le sens dérivé de « tête ».

- bourriche, bourrichon: bourriche est un autre mot argotique pour la tête, par analogie de forme avec un 'panier oblong.... servant au transport du gibier ou de

\footnotetext{
${ }^{4}$ Voir ce terme plus loin dans le texte.

${ }^{5}$ Alfred Delvau utilise l'orthographe bobêchon (avec un ê) et non bobéchon (avec un é).
} 
la marée...' d'après le Tlfi (consulté 06/20), qui mentionne bourriche d'huîtres et retient pour datation 1846, ce que confirme l'examen du tableau $1^{6}$. Pour bourrichon le Tlfi propose la date de 1860 pour le sens populaire «tête» de bourrichon dérivé de bourriche et cite l'expression se monter le bourrichon que Lucien Rigaud indique aussi (RIGAUD, 1888 : 57). Alfred Delvau mentionne de même le terme (DELVAU, $1867: 56$ ), mais ne retient pas bourriche (cf. le tableau 1).

- boussole: c'est par métonymie que l'on passe de l'instrument de navigation à la désignation populaire et argotique de la partie du corps, qui est le siège du jugement, de la raison, à savoir la tête. Ce terme est relevé par Alfred Delvau dès 1867 (DelvaU, $1867: 57$ ) et l'est encore en 1901 (Rossignol, $1901:$ 19) et en 1907 (HAYARD, $1907: 11$ ).

- cabèche: tout comme caboche (cf. ci-après) ce lexème signifie «tête » en langue familière et argotique; il s'agit d'un emprunt à l'espagnol cabeza, même sens. Il est seulement répertorié par deux des ouvrages (cf. le tableau 1) : (LA RUE, 1894 : 7) et (HAYARD, 1907 : 12). Cabèche est utilisé en 1888 par l'Abbé G. Moreau dans l'expression couper la cabèche, « couper la tête » $1888^{7}$.

- caboche : ce terme appartenant aux registres familier et argotique de la langue est présent dans 7/17 des glossaires et dictionnaires de l'étude, alors que cabèche ne l'est que dans 2/17, et il est noté dès 1849 (HALBERT D'ANGERS, 1849:11), cabèche ne l'étant qu'à compter de 1894 (LA RUE, 1894 : 7) (cf. ci-dessus). Caboche est attesté dès le XII ${ }^{\mathrm{e}}$ siècle sous la forme caboce avec deux sens ("Tête; grosse tête "; « tête dure et peu intelligente, tête obstinée ; forte tête, personne au caractère difficile ») et caboche date du XIII ${ }^{\mathrm{e}}$ siècle ${ }^{8}$.

- cafetière : ce substantif féminin désigne la tête en argot, vraisemblablement par analogie de forme avec l'appareil servant à préparer le café. On le trouve dans Lucien Rigaud (1888: 69), Jean La Rue (1894:8) et Gustave-Armand Rossignol $(1901: 21)$.

- caillou : ce mot utilisé pour un morceau de pierre de petite taille permet lui aussi par analogie de forme (ou par métaphore d'après le Tlfi (consulté 06/20)) de désigner la tête en langue populaire / argotique, plus particulièrement une tête chauve. Les ouvrages de Jean La Rue (1894: 8), Charles Virmaître (1894: 53) et Gustave-Armand Rossignol (1901:21) ont une entrée caillou, « tête ».

- caisson : pour ce lexème c'est par métaphore à partir du sens premier de 'caisse plus ou moins grande servant, en tant que contenant, à divers usages techniques' qu'est obtenu le sens " tête » en langue populaire. Caisson est relevé dès 1867 par Alfred Delvau (1867: 67), qui en attribue l'origine à l'argot des soldats. Il est ensuite mentionné par Lucien Rigaud (1888: 70) et Jean La Rue (1894 : 8). L'expression se faire sauter le caisson, « se suicider » est mentionnée par Alfred Delvau : 'se brûler la cervelle' (1867: 68) et par Lucien Rigaud: 'se faire sauter la cervelle' en précisant 'avec une arme à feu' (1888: 70).

\footnotetext{
${ }^{6}$ L'intérieur des prisons (1846) est le seul ouvrage parmi les 17 retenus, qui mentionne bourriche, tête.

${ }^{7}$ Abbé G. Moreau, Souvenirs de la Petite et de la Grande Roquette, tome II, p. 354.

${ }^{8}$ Caboche est relevé dans Le Roman de Troie de Benoit de Sainte-Maure (vers 1160-1170).
} 
- calebasse : ce mot a pour sens dérivé ${ }^{9}$ « tête » en langue argotique et populaire, ce qu'indique le Tlfi (consulté 06/20); il est relevé dès 1829 par un des ouvrages retenus pour la présente étude (BRAS-DE-FER, 1829:10 ${ }^{10}$ ) et la même année par Louis François Raban \& Émile Marco de Saint-Hilaire. Le sens «tête » est obtenu par allusion de forme pour Lorédan Larchey (1865: 51). Le terme est aussi mentionné avec ce sens par Alfred Delvau en tant que substantif employé en 'argot des faubouriens, qui ont trouvé une analogie quelconque entre l'os sublime et le fruit du baobab, presque aussi vides l'un que l'autre' (1867: 68) et Jean La Rue (1894 : 8). À noter que calebasses au pluriel désigne une 'gorge molle, qui promet plus qu'elle ne tient', des 'seins de fâcheuse apparence' (DELVAU, 1867 : 68, 468) ; c'est dans ce cas un synonyme de tétasses.

- ciboulot : en argot et langue populaire il s'agit du diminutif en -ot de ciboule $e^{11}$ ("plante condimentaire vivace employée dans les assaisonnements pour son goût qui s'apparente à celui de l'oignon' (Tlfi, 06/20)), "tête » par analogie de forme avec le bulbe de cette plante herbacée. Le mot ciboulot est une entrée dans 3/17 des ouvrages : Jean La Rue (1894: 9), Charles Virmaître (1894: 329) et GustaveArmand Rossignol (1901: 28). Charles Virmaitre mentionne (1894: 329) l'existence en argot du peuple de 'son ciboulot est vidé' à propos d'une personne qui a la tête vide. À mentionner aussi l'expression 'courir sur le ciboulot à qqn', « importuner sérieusement quelqu'un » (Tlfi, consulté 06/20).

- citron : c'est la forme ovoïde du fruit qui est prise en considération pour aboutir en argot par analogie de forme au sens «tête ». Lucien Rigaud est le premier à signaler ce lexème pour tête; il fait remonter l'origine du glissement de sens au jargon des voleurs (d'après sa typologie des formes langagières périphériques, non normées du XIX ${ }^{\mathrm{e}}$ siècle) (RIGAUD, 1888 : 100). 'Se presser le citron' avec les sens « faire des efforts désespérés pour essayer de réfléchir ou comprendre, se torturer l'esprit » est relevé par le Tlfi (consulté 06/20). Citron est aussi une entrée des dictionnaires de Gustave-Armand Rossignol (1901: 28) et Napoléon Hayard (1907: 13).

- cocarde : au total ce sont donc 3/17 ouvrages qui mentionnent ce lexème. Lorédan Larchey, qui est chronologiquement le premier parmi les 17 à noter ce terme, explique le sens «tête » ainsi: 'en prenant la coiffure pour la tête, on a dit taper sur la cocarde ou sur le pompon, pour : frapper sur la tête de quelqu'un'. (LARCHEY, $1865: 84$ ). Il s'agirait donc d'une synecdoque ${ }^{12}$. Cocarde est aussi relevé par Alfred Delvau (1867: 103) et Lucien Rigaud (1888: 102). Les trois auteurs indiquent la locution avoir sa cocarde pour être en état d'ivresse.

- coco : ce lexème est relevé par Alfred Delvau (1867 : 103), qui l'attribue à l'argot des faubouriens et rappelle les expressions coco déplumé pour une tête sans cheveux et redresser le coco, "porter la tête haute » (DELVAU, 1867 : 103-104).

\footnotetext{
${ }^{9}$ La définition de calebasse, qui appartient au vocabulaire de la botanique, est 'fruit du calebassier dont l'écorce, séchée, sert de récipient, d'objet de décoration' (Tlfi (consulté 06/20)).

${ }^{10}$ Bras-de-Fer mentionne aussi calebasse pour désigner une vieille tête (BRAS-DE-FER, 1829 : 51).

11 'Début XIX'e s. arg. des maçons, Puy-de-Dôme' (Tlfi (consulté 06/20)).

${ }^{12}$ C'est ce qu'indique l'explication de Lorédan Larchey dans la Revue anecdotique des lettres et des arts : 'Cocarde : tête. En prenant la partie pour le tout, on a dit taper sur la cocarde ou sur le pompon, pour frapper sur la tête de quelqu'un' (LARCHEY, 1858 : 458).
} 
Coco est aussi mentionné par Lucien Rigaud (1888 : 102) qui retient, quant à lui, la locution se monter le coco, «s'illusionner, se monter la tête ». Seuls ces deux auteurs ont inclus coco dans leur dictionnaire et on peut retenir la datation 1867 pour ce terme, puisqu'Alfred Delvau le relève dès cette année. Le Tlfi (consulté 06/20) date le sens dérivé du mot de 1847 en indiquant que ce sens 'pourrait être issu, p. métaph. due à l'aspect de la noix de coco fraîche qui présente trois trous la faisant ressembler à une tête humaine, de coco "croque-mitaine" à tête sphérique grossièrement figurée avec lequel on effraie les enfants'.

- coloquinte: 7/17 ouvrages (cf. le tableau 1) retiennent le mot coloquinte pour tête, ce dès 1839, puisqu'on le trouve dans le dictionnaire de Louis François Raban et Émile Marco de Saint-Hilaire (1829: 313) ${ }^{13}$. C'est par analogie de forme (LARCHEY, $1865: 87$ ) avec la coloquinte, qui est une plante grimpante de la famille des Cucurbitacées, que le sens dérivé « tête » est attribué en argot des faubouriens (DELVAU, 1867 : 106). Une coloquinte défraîchie est une tête de personne âgée sans cheveux (RIGAUD, 1888:105) et avoir une araignée dans la coloquinte signifie « avoir le cerveau fêlé » (BOUTMY, 1883: 72).

- fiole : la datation que l'on peut retenir est 1888, car seul le dictionnaire de Lucien Rigaud mentionne ce terme (1888:174), qui l'attribue à l'argot des voleurs. C'est par analogie de forme que l'on passe d'une 'petite bouteille de verre à goulot étroit' (Tlfi, consulté 06/20) au sens figuré populaire « tête ».

- hure : il s'agit de la 'tête du sanglier, du porc, $p$. ext. de certaines bêtes fauves et de poissons à tête allongée' (Tlfi, consulté en 06/20). L'usage familier de ce mot pour qualifier de manière dépréciative la tête d'une personne explique l'utilisation faite de ce substantif par la langue argotique pour désigner la tête, ce qu'attestent Charles Virmaître (1894: 146), Gustave-Armand Rossignol (1901: 59) et Napoléon Hayard (1907: 23).

Pour les deux vocables qui suivent, à savoir poire et pomme, c'est la référence qui est faite à la forme ronde de l'objet désigné, qui permet d'obtenir le sens dérivé de «tête ", tout comme pour balle, bille, binette, bobe, bobèche, bobéchon, bobine et boule $e^{14}$, entre autres.

- poire : le passage du sens premier de poire « fruit de forme oblongue » à celui de "tête" en langue populaire s'est opéré par analogie de forme du fait d'une allusion au visage de Louis Philippe que les caricaturistes du XIX ${ }^{\mathrm{e}}$ siècle représentent avec une tête en forme de poire (cf., entre autres, COLIN \& MEVEL, 1990 : 500). Le Tlfi précise aussi le glissement de sens par la locution se fendre la poire: 'l'origine de cette expression remonte à 1832 quand Charles Philipon réalisa une caricature de Louis Philippe avec une tête de poire. Depuis, poire a pris le sens argotique de visage' (consulté 06/20). 4/17 ouvrages retiennent ce mot (cf. le tableau 1), le premier étant celui de Lucien Rigaud, qui mentionne l'expression tambouriner la poire, "porter des coups au visage » (RIGAUD, 1888: 302). Pour Charles Virmaître 'se payer la tête de quelqu'un est synonyme de se payer sa poire (Argot du peuple)' (VIRMAITRE, 1894 : 226).

\footnotetext{
${ }^{13}$ L'expression 'Prends garde que Charlot ne joue à la boule avec ta coloquinte' (RABAN \& SAINTHiLAIRE (1829: 313)) signifie « prends garde que le bourreau ne te coupe la tête ».

${ }^{14}$ Voir plus haut dans le texte.
} 
- pomme : ce substantif, qui désigne la tête par analogie de forme avec le fruit ${ }^{15}$, est employé en argot des faubouriens pour Alfred Delvau (1867 : 386). Deux autres ouvrages citent ce terme plus tard, à savoir Jean La Rue (1894: 23) et GustaveArmand Rossignol (1901 : 87).

- sorbonne: en argot sorbonne signifie la tête 'en tant que siège de la pensée' (Tlfi, consulté 06/20). Voici ce qu'écrit Victor Hugo au sujet de la tête dans Le Dernier jour d'un condamné : "La tête d'un voleur a deux noms : la sorbonne, quand elle médite, raisonne et conseille le crime; la tronche, quand le bourreau la coupe» (HUGO, 1829 : 30). En 1881, dans Les Misérables, il reprend son texte en l'étoffant: "Le bandit a deux têtes, l'une qui raisonne ses actions et le mène pendant toute sa vie, l'autre qu'il a sur ses épaules le jour de sa mort; il appelle la tête qui lui conseille le crime, la sorbonne, et la tête qui l'expie, la tronche $»^{16}$. Le glissement de sens à partir de La Sorbonne, haut lieu du savoir apparaît clairement grâce aux deux citations de Victor Hugo. Le substantif sorbonne avec le sens de « tête » est indiqué par 10 des 17 ouvrages soumis à l'étude, ce dès 1829, année de la première édition du Dernier jour d'un condamné, dans les Mémoires d'un forçat ou Vidocq dévoilé (RABAN \& SAINT-HILAIRE, 1829 : 334-335 ${ }^{17}$ ).

- tirelire : le terme est mentionné avec le sens «tête" dès 1867 par Alfred DELVAU, qui précise qu'il s'agit d'un mot de l'argot des faubouriens indiquant 'où se mettent les économies de l'Étude et de l'Expérience' (DELVAU, 1867 : 471). Charles Virmaître, quant à lui, voit dans le sens dérivé de ce terme une 'allusion à la bouche qui représente exactement l'ouverture par laquelle on introduit les pièces de monnaie dans une tirelire' (VIRMAITRE, 1894 : 287). Ceci est plausible, puisqu'il existe en langue populaire les locutions bouche fendue comme une tirelire et bouche en tirelire pour une 'bouche aux lèvres minces et largement fendue' (Tlfi, consulté 06/20). Tirelire est aussi relevé par Gustave-Armand Rossignol (1901 : 104) et Napoléon Hayard (1907 : 37).

- toupie : un seul des 17 ouvrages contient ce terme argotique pour dire tête, celui d'Alfred Delvau, qui précise qu'on le trouve dans l'argot des faubouriens et cite la locution avoir du vice dans la toupie, "être très malin, savoir se tirer d'affaire » (DELVAU 1867 : 477). C'est bien le substantif féminin toupie désignant un 'jouet de forme conique, muni d'une pointe sur laquelle il se maintient en équilibre quand on lui imprime un mouvement de rotation' (Tlfi, consulté 06/20), qui par analogie de forme permet le glissement de sens.

- trombine : 2/17 ouvrages retiennent trombine, à savoir ceux d'Alfred Delvau (1867 : 485), qui attribue son origine à l'argot des faubouriens, et de Jean La Rue $\left(1894: 30^{18}\right)$. L'origine de ce terme du registre populaire est incertaine pour le $T l f i$ (consulté 06/20), qui note cependant sa valeur hypocoristique. Il s'agit d'un 'emprunt à l'italien trombina "petite trompe" 'qui aurait pu avoir, comme le français trompette, le sens de "nez, visage», mais l'ital. tromba semble avoir désigné seulement la trompe de l'éléphant, du moustique, de la mouche' (Tlfi,

\footnotetext{
${ }^{15}$ La tête étant une partie d'un tout, pomme désigne par métonymie une personne, un individu.

16 Victor Hugo, Les Misérables (1881) [QUATRIÈME PARTIE, L'IDYLLE RUE PLUMET ET L'ÉPOPÉE RUE SAINT-DENIS, LIVRE SEPTIÈME, L'argot, II, RACINES], p. 784.

${ }^{17}$ L'expression porter la sorbonne à Charlot, « aller à l'échafaud » y est mentionnée.

${ }^{18}$ Jean La Rue mentionne aussi le trompette pour tête (LA RUE, 1894 : 30 ).
} 
consulté 06/20). Pour Jacques Cellard et Alain Rey, ce lexème daté de 1836 est 'probablement [la] juxtaposition de trompe, pour «nez », et de bobine' (CELLARD \& REY, 1990 : 804).

- tronche: en observant le tableau on note que 14 des 17 glossaires et dictionnaires contiennent tronche; de 1807 à 1907 c'est le substantif populaire / argotique le plus mentionné pour désigner la tête. Il s'agit d'un terme ancien déjà relevé en 1596 avec le même sens dans La Vie Generevse des Mercelots... (PECHON DE RUBY, 1596 : 36), qui relève aussi comblette, désormais inusité, tout comme au $\mathrm{XIX}^{\mathrm{e}}$ siècle (aucun des 17 ouvrages ne le mentionne). Avant le glissement de sens opéré par la langue argotique tronche désigne un 'tronçon de bois gros et court' dans les dictionnaires des $\mathrm{XIX}^{\mathrm{e}}$ et $\mathrm{XX}^{\mathrm{e}}$ siècles. Le terme, forme féminine de tronc, qui provient du latin populaire trunca est initialement utilisé avec le sens de « bûche, poutre » (le Tlfi (consulté 06/20) indique 1298 comme datation pour ce sens).

Parmi l'ensemble des mots désignant la tête, pas seulement ceux retenus pour la présente étude, tronche est le seul qui est verlanisé dès la fin des années 1970, le début des années 1980, dans la variété langagière argotique que l'on nomme

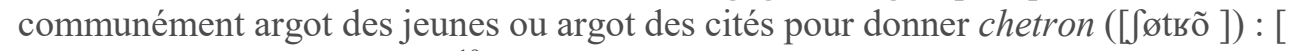

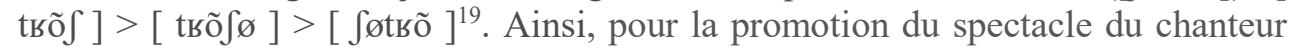
Renaud au Zénith de Paris en 1986 l'affiche indique 'Le retour de la chetron sauvage', preuve que le terme est bien présent dans le registre populaire / argotique du français.

\section{Conclusion}

Au terme de l'analyse il peut être établi que c'est le substantif tronche qui semble être le plus usité au XIX ${ }^{\mathrm{e}}$ siècle, si l'on se base sur le fait qu'il constitue une entrée dans le nombre le plus important, à savoir 14/17, des glossaires et dictionnaires retenus. Le deuxième score le plus élevé est celui de sorbonne :10/17.

Par ailleurs, l'ouvrage qui contient le plus de termes signifiant « tête » est celui de Lucien Rigaud (1888) avec 23/32 mots, suivi de celui de Jean La Rue (1894) (19/32) ; en troisième position on trouve Alfred Delvau (1867) (17/32). Plus loin derrière se situent en ordre décroissant Gustave-Armand Rossignol (1901) (12/32), Charles Virmaître (1894) (9/32) et Napoléon Hayard (1907) (8/32).

Si l'on compare les substantifs contenus d'une part dans les ouvrages de Lucien Rigaud (1888) et Jean La Rue (1894), d'autre part dans Colin \& Mével (1990), Cellard \& Rey (éd. Masson, 1990), on peut établir, qu'ils ont en commun 11 lexèmes pour "tête », à savoir balle, bille, binette, bobéchon, boule, cafetière, ciboulot, coco, pomme, sorbonne et tronche. Ces 11 substantifs sont donc retenus par les dictionnaires du $\mathrm{XIX}^{\mathrm{e}}$ mais le sont aussi par ceux du $\mathrm{XX}^{\mathrm{e}}$ siècle.

\footnotetext{
${ }^{19}$ Jean-Pierre Goudaillier, Comment tu tchatches ! - Dictionnaire du français contemporain des cités, Paris, Maisonneuve \& Larose, 1997 (4ème édition 2019), p. 95.
} 


\section{BIBLIOGRAPHIE}

ANONYME (1827), Dictionnaire d'argot ou Guide des gens du monde, pour les tenir en garde contre les mouchards, filous, filles de joie, et autres fashionables et petites-maîtresses de la même trempe. Par un monsieur comme il faut, expensionnaire de Ste-Pélagie, Paris, chez les marchands de nouveautés.

ANONYME (détenu anonyme), (1846), Dictionnaire renfermant les mots les plus usités dans le langage des prisons, in: L'intérieur des prisons - Réforme pénitentiaire, système cellulaire, emprisonnement en commun, Paris, Jules Labitte, p. 239-246 20.

BACHMANN Christian, BASIER Luc (1984), Le verlan argot d'école ou langue des Keums, in : Mots, n. 8, p. 169-187.

BRAS-DE-FER (1829), Nouveau Dictionnaire d'argot, par un Ex-Chef de brigade sous M. Vidocq; suivi de la chanson des galériens rapportée dans ses Mémoires. Ouvrage utile aux gens du monde, Paris, Guiraudet.

BouTMY Eugène (1883), Dictionnaire de l'argot des typographes, précédé d'une Monographie du Compositeur d'Imprimerie et suivi d'un choix de coquilles typographiques célèbres ou curieuses, Paris, C. Marpon et E. Flammarion éditeurs.

CELLARD Jacques, REY Alain (1990), Dictionnaire du français non conventionnel, Paris, Masson.

CLEMENS Jean-Joseph (1840), Dictionnaire d'argot, in : La Légende noire du bagne: Le Journal du forçat Clémens, Paris, (éditions Gallimard, Collection Découvertes Gallimard (Albums), 1992).

Colin Jean-Paul, Mevel Jean-Pierre (1990), Dictionnaire de l'argot, Paris, Larousse.

Delessale Georges (1896), Dictionnaire Argot-Français \& Français-Argot (Préface de Jean Richepin), Paris, Paul Ollendorff.

DELVAU Alfred, Dictionnaire érotique moderne par un professeur de langue verte, Freetown, Imprimerie de la Bibliomaniac Society, 1864.

Delvau Alfred (1867), Dictionnaire de la langue verte - Argots parisiens comparés, Deuxième édition entièrement refondue et considérablement augmentée, Paris, E. Dentu.

DELVAU Alfred (1889), Dictionnaire de la langue verte, nouvelle édition conforme à la dernière revue par l'Auteur, augmentée d'un supplément par Gustave FUSTIER, Paris, C. Marpon et E. Flammarion.

FRANCE Hector (1907), Dictionnaire de la langue verte, Paris, Librairie du Progrès. GOUDAILlier Jean-Pierre (1997), Comment tu tchatches! - Dictionnaire du français contemporain des cités, Paris, Maisonneuve \& Larose, (4 ${ }^{\text {ème }}$ édition 2019).

HALBERT D'ANGERS Arthur (1849), Le nouveau dictionnaire complet du Jargon de l'argot ou le langage des voleurs dévoilé contenant tous les mots usités reconnus et adoptés par eux, avec leurs explications et leurs définitions suivi des

${ }^{20}$ Lexique repris en partie dans Lazare Sainéan, Les sources de l'argot ancien, II (1912), p. 172-174. 
nouveaux genres de vols et escroqueries nouvellement employés par eux, et terminé par des chansons en français et en argot, Paris, Le Bailly.

HAYARD Napoléon (1907), Dictionnaire argot-français, Paris, Vve Léon Hayard.

Hugo Victor (1829), Le dernier jour d'un condamné, Paris, Charles Gosselin.

LARCHEY Lorédan (1858), Revue anecdotique des lettres et des arts, Paris, Librairie de la Rue de Seine, vol. VII.

LARCHE Lorédan (1865), Les Excentricités du langage, Cinquième édition, Paris, E. Lantu.

LECLAIR P. (1800), Dictionnaire d'argot, ou langage des voleurs, in : Histoire des brigands, chauffeurs et assassins d'Orgères, Chartres, imprimerie de Lacombe.

LA RUE Jean (1894), La langue verte. Dictionnaire d'argot et des principales locutions populaires, précédé d'une Histoire de l'Argot par Clément Casciani, Paris, P. Arnould.

M.D. (ouvrage recueilli par) (1844), Dictionnaire complet de l'Argot employé dans les Mystères de Paris. Ouvrage éminemment utile à toute personne honnête puisqu'il divulgue à la société les mots dont les filoux, voleurs, floueurs, chevaliers d'industrie composent leur conversation, Moulins, Imprimerie P.-A. Desrosiers.

MERLIN Léon (1888), La langue verte du troupier - dictionnaire d'argot militaire, Deuxième édition, Paris/Limoges, Henri Charles-Lavauzelle.

Moreau Abbé G. (1888), Souvenirs de la Petite et de la Grande Roquette, Paris, Librairie moderne.

PeChON De Ruby(1596), La Vie Generevse des Mercelots, Gvevz, et Boesmiens, contenans leur façon de viure, subtilitez \& Gergon, Lyon, chez Jean Julliéron.

RABAn Louis François, DE SAINT-HiLAIRE Émile Marco (1929), Glossaire d'argot, in: Mémoires d'un forçat ou Vidocq dévoilé (tome quatrième), Paris, H. Langlois Fils \& C ${ }^{\text {ie }}$, éditeurs, p. 299-338.

RIGAUD Lucien (1888), Dictionnaire de l'argot moderne, Paris, Paul Ollendorf.

ROSSIGNOL Gustave-Armand (1901), Dictionnaire d'argot (ARGOT-FRANÇAIS FRANÇAIS-ARGOT), Paris, Paul Ollendorff.

VIRMAITRE Charles (1894), Dictionnaire d'argot fin-de-siècle, Paris, A. Charles.

ZACCONE Pierre (1877), Histoire des bagnes jusqu'à nos jours, Paris, Victor Bunel. 